Research Paper

\title{
Postoperative pain assessment of robotic nipple-sparing mastectomy with immediate prepectoral prosthesis breast reconstruction: a comparison with conventional nipple- sparing mastectomy
}

\author{
Jiae Moon ${ }^{*}$, Jeea Lee ${ }^{2}$, Dong Won Lee ${ }^{3}$, Hye Sun Lee ${ }^{4}$, Da Jung Nam5 ${ }^{5}$ Min Jung Kim5, Na Young Kim ${ }^{1 凶}$, \\ Hyung Seok Park ${ }^{\circledR}$ \\ 1. Department of Anesthesiology and Pain Medicine, Anesthesia and Pain Research Institute, Yonsei University College of Medicine, Seoul, Republic of Korea. \\ 2. Department of Surgery, Yonsei University College of Medicine, Seoul, Republic of Korea. \\ Department of Plastic and Reconstructive Surgery, Yonsei University College of Medicine, Seoul, Republic of Korea. \\ 4. Department of Research Affairs, Biostatistics Collaboration Unit, Yonsei University College of Medicine, Seoul, Republic of Korea \\ 5. Department of Anesthesiology and Pain Medicine, Anesthesia and Pain Research Institute, National Health Insurance Service Ilsan Hospital, 100 Ilsan-ro, Ilsandong-gu, \\ Goyang, 10444, Republic of Korea. \\ *These authors contributed equally to this work. \\ $\triangle$ Corresponding authors: Na Young Kim, MD, PhD, Department of Anesthesiology and Pain Medicine, Anesthesia and Pain Research Institute, Yonsei University College \\ of Medicine, 50-1 Yonsei-ro, Seodaemun-gu, Seoul 03722, Republic of Korea. Office phone: 82-2-2228-2429; Fax: 82-2-312-7185; E-mail: knnyyy@yuhs.ac; Hyung Seok Park, \\ MD, PhD, Department of Surgery, Yonsei University College of Medicine, 50-1 Yonsei-ro, Seodaemun-gu, Seoul 03722, Republic of Korea. Office phone: 82-2-2228-2100; Fax: \\ 82-2-313-8189; E-mail: imgenius@yuhs.ac.
}

( ) The author(s). This is an open access article distributed under the terms of the Creative Commons Attribution License (https://creativecommons.org/licenses/by/4.0/). See http://ivyspring.com/terms for full terms and conditions.

Received: 2020.12.10; Accepted: 2021.04.04; Published: 2021.04.17

\begin{abstract}
Aims: Nipple-sparing mastectomy (NSM) is a surgical procedure for patients with breast cancer without nipple-areolar complex (NAC) involvement. Robotic NSM (RNSM) with immediate breast reconstruction has been recently introduced; however, reports regarding RNSM are still lacking. Therefore, this study aimed to evaluate the postoperative assessment with a focus on postoperative pain of RNSM with prepectoral immediate prosthesis breast reconstruction (IPBR) compared with conventional NSM (CNSM) in patients with breast cancer without NAC invasion.

Methods: This retrospective study included 81 patients who underwent RNSM $(n=40)$ or CNSM $(n=41)$ with prepectoral IPBR using direct-to-implant or tissue expander between January 2018 and June 2020. The primary endpoint was to compare postoperative pain intensity based on a numerical rating scale (NRS). The secondary endpoint was to evaluate the postoperative recovery profile, including postoperative nausea/vomiting (PONV) and complications.

Results: A statistical difference was observed in the resting NRS scores at $0-6$ postoperative hours between the RNSM and CNSM groups (3.2 \pm 1.5 versus $4.2 \pm 1.6$, respectively; Bonferroni corrected $P=0.005)$, however, no difference was shown at other time periods. Also, no between-group difference was found in the NRS scores for acting pain within 48 postoperative hours and the number of patients requiring additional analgesics.

Conclusions: Despite a statistical difference in the resting NRS scores during the early postoperative phase, the absence of any significant difference in the requirement of additional analgesics between the groups suggested that RNSM does not significantly attenuate postoperative pain intensity.
\end{abstract}

Key words: breast cancer; robotic; conventional; nipple-sparing mastectomy; postoperative pain

\section{Introduction}

Nipple-sparing mastectomy (NSM) is a surgical procedure performed in patients with breast cancer without the involvement of the nipple-areolar complex (NAC), to improve the cosmetic outcome and quality of life in women with early breast cancer or germline $B R C A 1 / 2$ mutation. In NSM, the skin envelope and NAC are preserved, and the glandular breast tissue is completely removed. NSM allows 
immediate breast reconstruction (IBR) using autologous tissue and/or implants [1]. Preservation of the NAC enhances the esthetic outcome and patient satisfaction without compromising oncologic safety [2]. However, dissecting and removing breast tissue through limited incisions is technically challenging. Some NSM incisions leave visible scars on the breast dome and cause distortion or malposition of the NAC. NAC-related necrosis also remains a major NSM complication [3,4].

Robotic NSM (RNSM) with IBR was first described by Toesca et al. [5] in October 2015 and has substantially contributed to overcoming the challenges of conventional NSM (CNSM) [6]. A highresolution ten-fold magnifying three-dimensional camera allows accurate visualization and better access to the surgical planes, which enables RNSM with IBR through a small axillary incision. The flexibility and sophisticated motion of robotic surgical systems increase the surgical accuracy in a limited space $[3,7]$. RNSM with a 2.5-6-cm axillary incision has excellent patient satisfaction and cosmetic results, considering the absence of scarring in the anterior breast. RNSM has low ischemic NAC complication rates, which may be attributed to the remote incision from the NAC and the vascular advantage allowed by sparing small vessels responsible for vascular supply to the nipple $[7,8]$.

Despite several limitations, including a longer duration of operation, higher cost, and lack of research on the long-term oncological outcomes, RNSM with IBR has been increasingly used worldwide given its definite advantage with respect to esthetics [6,8-11]. However, there are few studies on the postoperative outcomes of RNSM with IBR [8].

Thus, this study aimed to evaluate the postoperative parameters, particularly postoperative pain, of RNSM with prepectoral immediate prosthesis

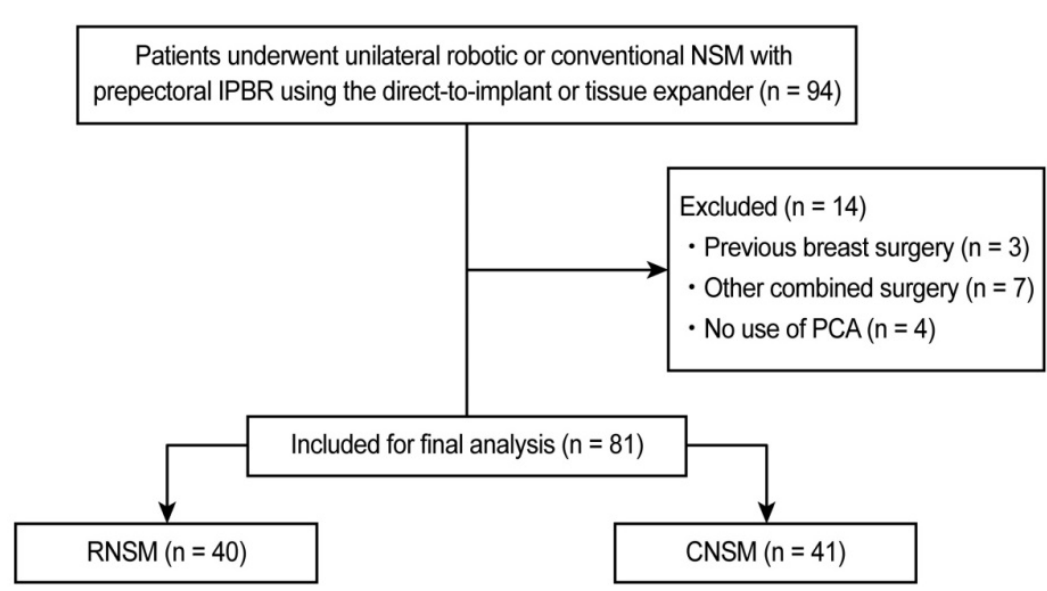

Figure 1. Consort flow diagram. NSM, nipple-sparing mastectomy; IPBR, immediate prosthesis breast reconstruction; PCA, patient-controlled analgesia; RNSM, robotic nipple-sparing mastectomy; CNSM, conventional nipple-sparing mastectomy. breast reconstruction (IPBR) using direct-to-implant (DTI) or tissue expander, compared with CNSM in patients with breast cancer without NAC invasion.

\section{Materials and methods}

\section{Patient population}

We identified the records of 94 consecutive patients with breast cancer who underwent unilateral RNSM or CNSM with prepectoral IPBR using DTI or tissue expander between January 2018 and June 2020 at Severance Hospital, Seoul, Korea. Three patients who had previously undergone breast surgery, seven patients who underwent other simultaneous surgeries, and four patients who were not administered intravenous patient-controlled analgesia (IV-PCA) for postoperative pain control were excluded. Consequently, 81 patients who underwent RNSM $(n=40)$ and CNSM $(n=41)$ were analyzed (Figure 1).

\section{Surgical procedures}

\section{RNSM}

Detailed procedures for RNSM have been previously described [3,9-11]. Briefly, a 2.5-6-cm linear mid-axillary incision was made below the axillary fossa. The ipsilateral arm was straightened with internal rotation and abduction and fixed above the head. A sentinel lymph node biopsy was performed through the incision; moreover, the working space beneath the skin flap or retromammary space was manually developed. Subsequently, a single-port device was inserted through the same incision for the gas-inflated technique and the robotic surgical system was docked. After carbon dioxide gas insufflation with 8$10 \mathrm{mmHg}$ for surgical field expander, the skin flap and/or retromammary space were dissected using the robotic surgical system. During the mastectomy, the sub-NAC tissue was resected to evaluate tumor cell involvement in a frozen section. Finally, the entire breast parenchyma was retrieved through the same incision, and prepectoral IPBR using DTI or tissue expander was performed by the plastic surgeons $[3,10]$.

\section{CNSM}

Various skin incisions such as inframammary fold incision, radial incision, and/or periareolar/ circumareolar incision were made. The skin flaps were developed along the superficial fascia superior to the lower 
clavicular border, medial to the ipsilateral sternal border, inferior to the rectus sheath, and posterior to the anterior latissimus dorsi muscle border. The entire breast gland was dissected from the pectoralis major muscle, including the pectoralis fascia. A frozen section for the sub-NAC tissue was intraoperatively obtained. Through the same incision, sentinel lymph node biopsy using navigator and/or axillary lymph node dissection was performed. After the mastectomy, prepectoral IPBR using tissue expander or implant was performed by the plastic surgeons [12].

\section{IPBR}

IPBR after mastectomy was performed using DTI or tissue expander in the prepectoral space. The implant or tissue expander was completely wrapped with acellular dermal matrix (ADM) before insertion. Patients undergoing autologous reconstruction or subpectoral prosthesis were excluded from the current study.

\section{Anesthetic procedures}

General anesthesia was administered following our institution's conventional protocol. After the patient arrived in the operating room, all devices monitoring oxygen saturation, electrocardiography, noninvasive arterial blood pressure, and bispectral index (BIS), were utilized to evaluate the patient. Intravenous (IV) injection of $0.1 \mathrm{mg}$ glycopyrrolate was administered as premedication. Subsequently, anesthesia was induced using propofol (1-1.5 mg/ kg), rocuronium $(0.6 \mathrm{mg} / \mathrm{kg})$, and remifentanil (0.05-0.1 $\mu \mathrm{g} / \mathrm{kg})$. Mechanical ventilation was initiated with an inspiration to expiration (I:E) ratio of $1: 2$, positive end-expiratory pressure of $5 \mathrm{cmH}_{2} \mathrm{O}$, tidal volume of $6-8 \mathrm{ml} / \mathrm{kg}$, and respiratory rate of $8-14$ frequency/ min to maintain an end-tidal carbon dioxide of 35-42 $\mathrm{mmHg}$. Anesthesia was maintained using sevoflurane or desflurane (0.8-1 age-adjusted minimum alveolar concentration) and remifentanil at $0.03-0.1 \mu \mathrm{g} / \mathrm{kg} /$ min. To maintain a constant anesthetic depth, the BIS was continuously monitored, with a target range of 40-60.

\section{Postoperative pain management}

Prior to the end of the surgery, $1 \mu \mathrm{g} / \mathrm{kg}$ of fentanyl (Hana Pharm, Seoul, Korea) and $0.3 \mathrm{mg}$ of ramosetron (Nasea ${ }^{\circledR}$, Astellas Pharma Korea, Seoul, Korea) were concurrently administered to relieve postoperative pain and nausea/vomiting, respectively. All patients received an IV-PCA device (Anapa plus; E-HWA Biomedics, Seoul, Korea), programmed to $2 \mathrm{~mL} / \mathrm{h}$ for background infusion, a demand volume of $0.5 \mathrm{~mL}$, lock-out interval of 15 minutes, with a total volume of $100 \mathrm{~mL}$. The PCA regimen comprised $15 \pm 2 \mu \mathrm{g} / \mathrm{kg}$ of fentanyl and 0.3 $\mathrm{mg}$ of ramosetron, which were mixed with normal saline to achieve a total volume of $100 \mathrm{~mL}$. Additionally, patients in both groups received $1 \mathrm{~g}$ IV paracetamol (profa ${ }^{\circledR}$, Dai han Pharm, Seoul, Korea) at 8-h intervals for 5 days, and one Mypol $^{\circledR}$ tablet (codeine phosphate $10 \mathrm{mg}$ plus ibuprofen $200 \mathrm{mg}$, Sung-won Adcock Pharm, Seoul, Korea) at 8-h intervals until discharge.

\section{Postoperative pain assessment}

Data regarding postoperative pain was obtained from an electronic medical database, which was recorded by a PCA management team comprising two qualified nurses. Resting pain was defined as pain while at rest or staying still; acting pain was defined as pain during movement, posture change, or coughing. All eligible patients were informed on how to rate their pain intensity using the numerical rating scale (NRS; 0, no pain; 10, worst pain possible) in the pre-anesthetic room [13]. After the patients were moved to the post-anesthetic care unit (PACU) and had emerged from anesthesia, the recovery nurses assessed their NRS scores. The patients were instructed about the use of the PCA device, and were encouraged to push the button whenever they experienced pain. In patients who experienced sustained pain with a resting NRS score $\geq 4,50 \mu \mathrm{g}$ IV fentanyl was administered as an additional rescue analgesic. After the patients were transferred to the admission room, postoperative resting and acting NRS score assessments were performed at 0-6, 6-24, and 24-48 postoperative hours. In patients who suffered from the prolonged pain with a resting NRS score of $\geq 4$ in the admission room, $50 \mathrm{mg}$ tridol (Tramadol HCL®, Yuhan. Co., Seoul, Korea) was administered as a rescue analgesic.

\section{Postoperative nausea and vomiting (PONV) and complications}

Postoperative management during hospitalization was left at the discretion of the Yonsei Breast Cancer Center team. PONV was assessed on a 4-point NRS $(0-3 ; 0=$ none, $1=$ mild, $2=$ moderate, $3=$ severe). All patients received $0.3 \mathrm{mg}$ IV ramostron at 24-h intervals for 3 days. Rescue antiemetics were administered when severe nausea or vomiting developed, or upon request from the patients. Metoclopramide (Macperan ${ }^{\circledR}$, Dong Wha Pharm. Co., Ltd., Seoul, Korea) $10 \mathrm{mg}$ was administered as a first-line rescue antiemetic. Patients with persistent and refractory PONV received $0.3 \mathrm{mg}$ IV ramosetron. The incidence of postoperative complications was assessed for up to 90 postoperative days. Major complications included implant loss, nipple necrosis, 
and/or mastectomy skin flap necrosis requiring surgical treatment. Mild complications included seroma, hematoma, minor infection, wound dehiscence, and nipple or skin flap necrosis with conservative management.

\section{Data collection}

Demographic and preoperative characteristics, including age, body mass index (BMI), American Society of Anesthesiologists physical status, smoking history, menopause status, family history of breast cancer, and neoadjuvant chemotherapy were assessed. Regarding perioperative and surgical characteristics, duration of anesthesia and operation, blood loss, dose of remifentanil administered, dose of fentanyl combined with PCA, surgical incision length, mastectomy specimen weight, location, reconstruction type, reconstruction implant volume, and adjuvant treatment were assessed. Further, pathologic variables were collected. Additionally, postoperative profiles, including postoperative hospital stay, PONV, and complications were evaluated. Postoperative NRS scores to determine the maximum resting and acting pain were assessed in the PACU at 0-6, 6-24, and 24-48 postoperative hours. Furthermore, the number of patients requiring additional analgesics for up to postoperative 48 hours were assessed.

\section{Statistical analysis}

Continuous variables are presented as mean \pm standard deviation (SD) while categorical variables are presented as the number of patients (percentage). Between-group differences in the continuous and categorical variables were analyzed using the Student's t-test and the Chi-square test/Fisher's exact test, respectively. To determine the group and time effects for repeated-measure continuous and categorical variables, linear mixed model analysis and generalized estimating equations were performed, respectively. Bonferroni correction was applied to adjust for multiple comparisons in post-hoc analyses. $\mathrm{P}<0.05$ was considered statistically significant. All analyses were conducted using SAS version 9.4 (Cary, NC).

\section{Results}

Table 1 demonstrates the clinicopathologic factors of the patients selected for this retrospective analysis. The average BMI in the RNSM group was lower than that in the CNSM group by $1.7 \mathrm{~kg} / \mathrm{m}^{2}(22.2$ \pm 3.5 vs. $23.9 \pm 3.6 \mathrm{~kg} / \mathrm{m}^{2}, P=0.030$ ), and no between-group differences were observed regarding other variables. Table 2 summarizes the intraoperative and surgical variables. Duration of anesthesia and operation were significantly longer in the RNSM group than in the CNSM group (both $P<$ $0.001)$. There was no between-group difference in the dose of fentanyl used for PCA $(15.0 \pm 1.8 \mu \mathrm{g} / \mathrm{kg}$, RNSM group; $14.6 \pm 1.6 \mu \mathrm{g} / \mathrm{kg}, \mathrm{CNSM}$ group). The surgical incision length in the RNSM group (4.4 \pm 0.7 $\mathrm{cm}$ ) was significantly shorter than that in the CNSM group $(8.8 \pm 2.2 \mathrm{~cm} ; P<0.001)$.

Table 1. Clinicopathologic factors of patients selected for the analysis

\begin{tabular}{|c|c|c|c|}
\hline & $\operatorname{RNSM}(\mathrm{n}=40)$ & $\operatorname{CNSM}(\mathrm{n}=41)$ & $P$ value \\
\hline \multicolumn{4}{|l|}{ Patients characteristics } \\
\hline Age, years & $46 \pm 8$ & $49 \pm 10$ & 0.177 \\
\hline Body mass index, $\mathrm{kg} / \mathrm{m}^{2}$ & $22.2 \pm 3.5$ & $23.9 \pm 3.6$ & $0.030^{*}$ \\
\hline ASA physical status & & & 0.381 \\
\hline I & $19(48 \%)$ & $16(39 \%)$ & \\
\hline II & $19(48 \%)$ & $19(46 \%)$ & \\
\hline III & $2(5 \%)$ & $6(15 \%)$ & \\
\hline Smoking history & & & 0.836 \\
\hline Non-smoker & $37(93 \%)$ & $36(88 \%)$ & \\
\hline Ex-smoker & $2(5 \%)$ & $4(10 \%)$ & \\
\hline Current-smoker & $1(2 \%)$ & $1(2 \%)$ & \\
\hline Menopause status & & & 0.268 \\
\hline Premenopausal & $28(70 \%)$ & $25(64 \%)$ & \\
\hline Postmenopausal & $10(25 \%)$ & $14(36 \%)$ & \\
\hline Perimenopausal & $2(5 \%)$ & $0(0 \%)$ & \\
\hline Neoadjuvant chemotherapy & $5(13 \%)$ & $11(27 \%)$ & 0.105 \\
\hline \multicolumn{4}{|l|}{ Pathologic characteristics } \\
\hline Pathologic tumor size, $\mathrm{cm}$ & $1.6 \pm 1.3$ & $1.8 \pm 1.1$ & 0.420 \\
\hline Multicentric/multifocal lesion & $19(48 \%)$ & $16(39 \%)$ & 0.441 \\
\hline Lymph node procedure & & & 0.712 \\
\hline SLNB only & $37(93 \%)$ & $36(88 \%)$ & \\
\hline SLNB then ALND & $3(8 \%)$ & $5(12 \%)$ & \\
\hline Pathologic staging, pT & & & 0.283 \\
\hline Tis & $6(15 \%)$ & $9(22 \%)$ & \\
\hline $\mathrm{T} 1$ & $28(70 \%)$ & $21(51 \%)$ & \\
\hline $\mathrm{T} 2$ & $6(15 \%)$ & $9(22 \%)$ & \\
\hline T0 & $0(0 \%)$ & $2(5 \%)$ & \\
\hline Pathologic staging, $\mathrm{pN}$ & & & 0.432 \\
\hline No & $37(93 \%)$ & $36(88 \%)$ & \\
\hline N1 & $2(5 \%)$ & $5(12 \%)$ & \\
\hline N2 & $0(0 \%)$ & $0(0 \%)$ & \\
\hline N3 & $1(3 \%)$ & $0(0 \%)$ & \\
\hline Histopathologic grade & & & 0.300 \\
\hline G1 & $13(33 \%)$ & $10(25 \%)$ & \\
\hline G2 & $23(57 \%)$ & $21(53 \%)$ & \\
\hline G3 & $4(10 \%)$ & $9(23 \%)$ & \\
\hline HER2 status & & & 0.173 \\
\hline Negative & $28(70 \%)$ & $24(59 \%)$ & \\
\hline Positive & $11(28 \%)$ & $11(27 \%)$ & \\
\hline Not available & $1(3 \%)$ & $6(15 \%)$ & \\
\hline Estrogen receptor status & & & 0.446 \\
\hline Negative & $7(18 \%)$ & $10(24 \%)$ & \\
\hline Positive & $33(83 \%)$ & $31(76 \%)$ & \\
\hline Progesterone receptor status & & & 0.352 \\
\hline Negative & $9(23 \%)$ & $13(32 \%)$ & \\
\hline Positive & $31(78 \%)$ & $28(68 \%)$ & \\
\hline Ki 67 & & & 0.223 \\
\hline Low $(<14 \%)$ & $20(50 \%)$ & $26(63 \%)$ & \\
\hline High $(\geq 14 \%)$ & $20(50 \%)$ & $15(37 \%)$ & \\
\hline \multicolumn{4}{|c|}{$\begin{array}{l}\text { Notes: Data are presented as mean } \pm \text { standard deviation or number of patients } \\
\text { (proportion). }{ }^{*} P<0.05 \text {. }\end{array}$} \\
\hline \multicolumn{4}{|c|}{$\begin{array}{l}\text { Abbreviation: RNSM, robotic nipple sparing mastectomy; CNSM, conventional } \\
\text { nipple-sparing mastectomy; ASA, American Society of Anesthesiologists; SLNB, } \\
\text { sentinel lymph node biopsy; ALNB, axillary lymph node biopsy; HER2, human } \\
\text { epidermal growth factor receptor 2; Tis, carcinoma in situ. }\end{array}$} \\
\hline
\end{tabular}


Table 2. Perioperative and surgical characteristics

\begin{tabular}{|c|c|c|c|}
\hline & $\operatorname{RNSM}(n=40)$ & CNSM $(n=41)$ & $P$ value \\
\hline Anesthesia time, $\min$ & $331 \pm 74$ & $241 \pm 43$ & $<0.001^{*}$ \\
\hline Operation time, min & $279 \pm 63$ & $207 \pm 46$ & $<0.001^{*}$ \\
\hline Blood loss & & & 0.057 \\
\hline$\leq 100 \mathrm{~mL}$ & $34(85 \%)$ & $40(98 \%)$ & \\
\hline$>100 \mathrm{~mL}$ & $6(15 \%)$ & $1(2 \%)$ & \\
\hline Administered remifentanil $(\mu \mathrm{g} / \mathrm{kg} / \mathrm{hr})$ & $0.047 \pm 0.010$ & $0.047 \pm 0.010$ & 0.998 \\
\hline $\begin{array}{l}\text { Fentanyl amounts mixed in PCA } \\
(\mu \mathrm{g} / \mathrm{kg})\end{array}$ & $15.0 \pm 1.8$ & $14.6 \pm 1.6$ & 0.221 \\
\hline Incision length, $\mathrm{cm}$ & $4.4 \pm 0.7$ & $8.8 \pm 2.2$ & $<0.001^{*}$ \\
\hline Specimen weight, $g$ & $388.7 \pm 169.5$ & $421.4 \pm 176.0$ & 0.400 \\
\hline Location & & & 0.144 \\
\hline Right & $25(63 \%)$ & $19(46 \%)$ & \\
\hline Left & $15(38 \%)$ & $22(54 \%)$ & \\
\hline Type of reconstruction & & & 0.195 \\
\hline Direct-to-implant & $32(80 \%)$ & $37(90 \%)$ & \\
\hline Tissue expander insertion & $8(20 \%)$ & $4(10 \%)$ & \\
\hline Volume of reconstruction implant, $\mathrm{mL}$ & $366 \pm 111$ & $357 \pm 110$ & 0.718 \\
\hline \multicolumn{4}{|c|}{ Adjuvant treatment } \\
\hline Radiation therapy & $5(13 \%)$ & $8(20 \%)$ & 0.390 \\
\hline Chemotherapy & $10(25 \%)$ & $12(29 \%)$ & 0.666 \\
\hline Endocrine therapy & $32(80 \%)$ & $26(67 \%)$ & 0.180 \\
\hline
\end{tabular}

Notes: Data are presented as mean \pm standard deviation or number of patients (proportion). ${ }^{*} P<0.05$.

Abbreviation: RNSM, robotic nipple-sparing mastectomy; CNSM, conventional nipple-sparing mastectomy; PCA, patient-controlled analgesia.
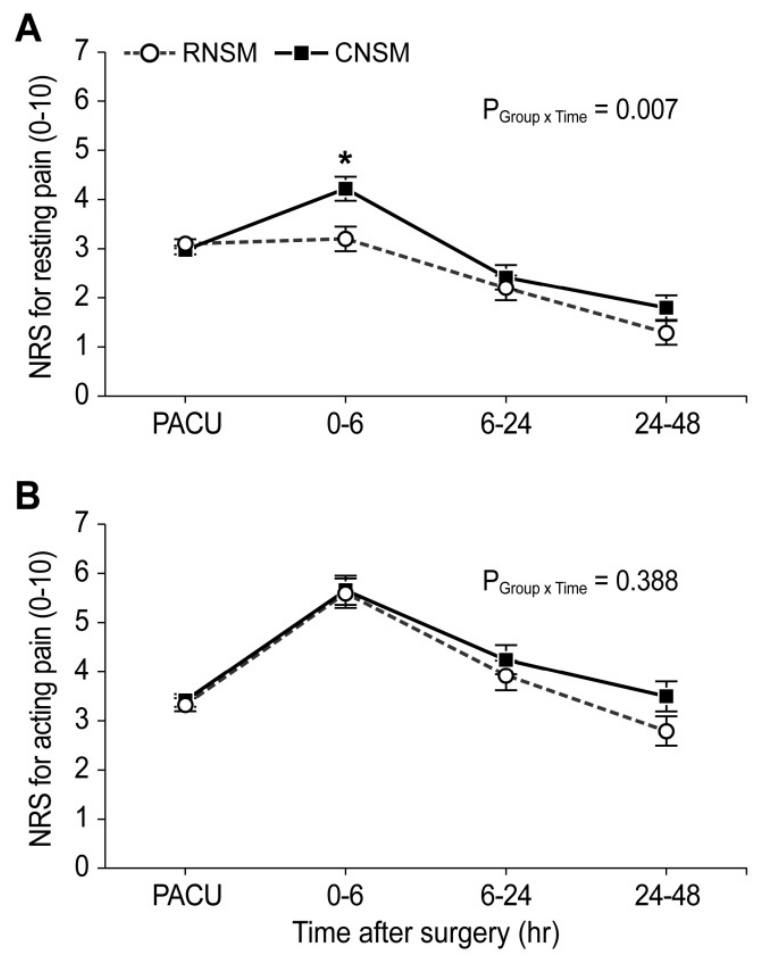

Figure 2. Resting (A) and acting (B) pain intensity in the RNSM and CNSM groups during 48 postoperative hours. NRS, numerical rating scale; RNSM, robotic nipple-sparing mastectomy; CNSM, conventional nipple-sparing mastectomy; PACU, post-anesthesia care unit. *Bonferroni corrected $P=0.005$ compared with CNSM.

Figure 2 presents the resting and acting pain intensity. The linear mixed model analysis revealed significant between-group differences in the resting NRS score $(P$ Group $x$ Time $=0.007)$ (Figure 2A). After the post-hoc analysis, a statistical difference was observed in the resting NRS scores at 0-6 postoperative hours between the RNSM and CNSM groups $(3.2 \pm 1.5$ versus $4.2 \pm 1.6$, respectively; Bonferroni corrected $P=$ 0.005); however, no difference was shown at other time periods (PACU, 6-24, and 24-48 postoperative hours). Additionally, there was no between-group difference in the NRS scores for acting pain up to 48 postoperative hours (Figure 2B), and in the number of patients requiring additional analgesics (Figure 3 ).

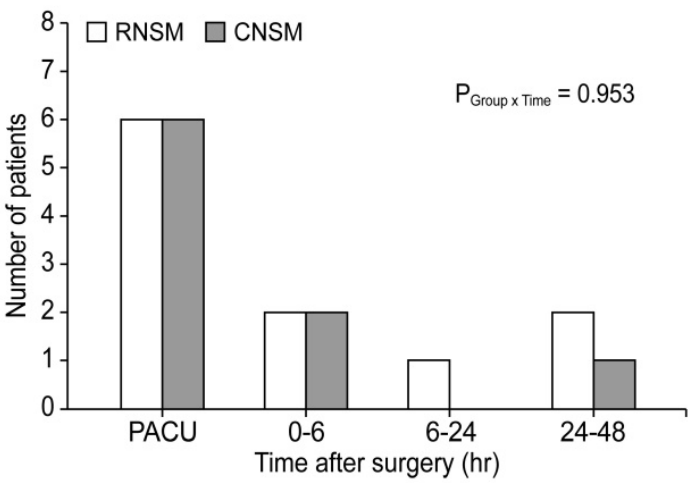

Figure 3. Number of patients who required additional analgesics during the first 48 postoperative hours. RNSM, robotic nipple sparing mastectomy; CNSM, conventional nipple sparing mastectomy; PACU, post-anesthesia care unit.

Table 3. Postoperative profile

\begin{tabular}{|c|c|c|c|}
\hline & $\operatorname{RNSM}(n=40)$ & $\operatorname{CNSM}(n=41)$ & $P$ value \\
\hline Postoperative hospital stays, days & $9.2 \pm 2.7$ & $7.1 \pm 2.0$ & $<0.001^{*}$ \\
\hline $\begin{array}{l}\text { Postoperative hospital stays, days } \\
\text { (recent } 20 \text { cases) }\end{array}$ & $7.8 \pm 1.9$ & $7.0 \pm 2.2$ & 0.252 \\
\hline \multicolumn{4}{|l|}{ Nausea (severe/moderate/mild/none) } \\
\hline At PACU & $6 / 2 / 0 / 32$ & $5 / 2 / 0 / 34$ & 0.906 \\
\hline 0-6 hours & $8 / 5 / 1 / 26$ & $10 / 7 / 2 / 22$ & 0.747 \\
\hline 6-24 hours & $4 / 6 / 5 / 25$ & $7 / 4 / 1 / 29$ & 0.262 \\
\hline \multicolumn{4}{|l|}{ Vomiting } \\
\hline At PACU & $2(5 \%)$ & $3(7 \%)$ & $>0.999$ \\
\hline 0-6 hours & $6(15 \%)$ & $12(29 \%)$ & 0.123 \\
\hline 6-24 hours & $2(5 \%)$ & $6(15 \%)$ & 0.264 \\
\hline \multicolumn{4}{|l|}{ Postoperative complication } \\
\hline Seroma & $2(5 \%)$ & $2(5 \%)$ & $>0.999$ \\
\hline Hematoma & $0(0 \%)$ & $2(5 \%)$ & 0.494 \\
\hline Wound dehiscence & $1(3 \%)$ & $4(10 \%)$ & 0.359 \\
\hline Infection & $3(8 \%)$ & $3(7 \%)$ & $>0.999$ \\
\hline \multicolumn{4}{|l|}{ Nipple necrosis } \\
\hline Surgical Tx & $0(0 \%)$ & $1(2 \%)$ & $>0.999$ \\
\hline Conservative $\mathrm{Tx}$ & $5(13 \%)$ & $5(12 \%)$ & $>0.999$ \\
\hline \multicolumn{4}{|l|}{ Mastectomy skin flap necrosis } \\
\hline Surgical Tx & $2(5 \%)$ & $6(15 \%)$ & 0.264 \\
\hline Conservative $\mathrm{Tx}$ & $1(3 \%)$ & $1(2 \%)$ & $>0.999$ \\
\hline
\end{tabular}

Table 3 presents the postoperative recovery profiles, including PONV and postoperative complications. In the analysis of the last 20 cases in each group, no difference was observed between the two groups in the average length of postoperative hospital stay $(P=0.252)$. Moreover, there was no between-group difference in the number of patients who had PONV and complications up to 90 postoperative days. No patient had unrecoverable complications. 


\section{Discussion}

This study analyzed postoperative pain in RNSM with prepectoral IPBR compared with CNSM, which showed a statistical difference in resting NRS scores between the two groups; however, the difference was transient and observed only during 0-6 postoperative hours. Further, no between-group difference was observed in the number of patients requiring additional analgesics, the incidence of PONV, and postoperative complications.

Approximately $25 \%-60 \%$ of patients present with persistent pain after breast cancer surgery, which is a major clinical issue [14,15]. It leads to chronic pain in a substantial number of patients, which decreases the quality of life and deteriorates their physical functions $[16,17]$. There may be multifactorial risk factors for this pain, including patient-related and treatment-related risk factors, such as the type of procedure performed [15]. Therefore, there is a need for surgical approaches that alleviate postoperative pain to improve the postoperative outcome in patients. Minimally invasive robotic approaches have been demonstrated to reduce postoperative pain for various surgeries $[18,19]$. Given the rapid expander of robotic approaches for breast procedures, there have been several reports regarding the feasibility and advantages of RNSM with IBR [4-8]. However, no evaluation regarding the postoperative pain assessment in RNSM compared with CNSM has been performed.

In this study, a statistical difference was observed in the resting NRS scores at 0-6 postoperative hours between the RNSM and CNSM groups (3.2 \pm 1.5 versus $4.2 \pm 1.6$, respectively; Bonferroni corrected $P=0.005$ ); however, which might be subjective depending on the patients, and difference was transient and observed only during the 0-6 postoperative hours. Additionally, no difference was observed at other time periods (PACU, 6-24, and 24-48 postoperative hours), which may suggest that some degree of pain control was achieved through routine IV PCA. This might negate the minor difference in pain associated with skin flap incision and length (4.4 cm versus $8.8 \mathrm{~cm} ; \sim 4.4-\mathrm{cm}$ difference) between the two types of surgeries. To control for other factors that may affect pain intensity, we only included patients who underwent unilateral NSM and prepectoral IPBR, which involves wrapping the prosthesis in a material such as ADM and placing it behind the skin flap. This approach is less invasive, more cosmetically effective, and less painful since pectoralis major muscle (PMM) dissection is not required. In contrast, subpectoral IPBR involves placement of the prosthetic device in the submuscular pocket behind the PMM $[20,21]$. However, no difference was observed between the two groups regarding acting pain intensity during 48 postoperative hours and the number of patients who required additional analgesics.

Between-group differences were observed in the demographics in terms of incision length, BMI, operation time, and hospital stay. BMI in the RNSM group was lower than that in the CNSM group by 1.7 $\mathrm{kg} / \mathrm{m}^{2}\left(22.2 \pm 3.5\right.$ vs. $23.9 \pm 3.6 \mathrm{~kg} / \mathrm{m}^{2}$, respectively $)$, which was statistically, though not clinically, different between the groups, since there was no between-group difference in the breast volume. A smaller incision length, longer duration of operation, and higher medical cost were specific RNSM features, which is consistent with the findings of a previous study [3,8]. In this study, the length of hospital stay in the RNSM group was 2 days longer than that in the CNSM group, which might be attributed to the preference of both patients and plastic surgeons for a slightly longer hospital stay in the early phase of RNSM to prioritize safety in an innovative surgical procedure. However, after the initial experience in RNSM, this difference in the length of hospital stay disappeared, as observed in the results of the analysis of length of hospital stay among the last 20 patients in each group $(7.8 \pm 1.9$ versus $7.0 \pm 2.2$ days, $P=0.252)$. Additionally, there was no between-group difference in the number of patients who presented with PONV.

With regard to postoperative complications, there was no difference between the two groups in the present study, which was consistent with the findings of Lai HW et al. [8]. However, in a recent report by Lee et al., RNSM showed significantly lower postoperative nipple necrosis and high-grade postoperative complications rates, [22] a discrepancy that appears to be due to a difference in the study population. The previous report by Lee et al. from our institution included $>200$ cases of robotic and conventional mastectomy, including autologous breast reconstruction, whereas the current study included only NSM with implant-based reconstruction. All patients who experienced complications in this study recovered completely without any other severe complications.

To our knowledge, this is the first study comparing RNSM versus CNSM with a focus on postoperative pain. However, this study has several limitations, including its retrospective nature, small sample size, and missing information, such as the total consumed dose of analgesics per time unit, frequency and dose of bolus on demand, and chronic pain assessment after 48 hours. We could only evaluate the analgesia quality by comparing the pain intensity based on the NRS scores and the number of patients requiring rescue analgesic as a surrogate. To 
overcome those limitations and clarify the findings of this study, further large-scale prospective trials are needed. Nonetheless, our findings are clinically significant to the existing literature.

\section{Conclusions}

Despite a statistical difference in the resting NRS scores during the early postoperative phase, the absence of any significant difference in the requirement of additional analgesics between the groups suggested that RNSM does not significantly attenuate postoperative pain intensity in patients with breast cancer without NAC invasion. Further prospective trials are needed to clarify this.

\section{Abbreviations}

NSM: nipple-sparing mastectomy; NAC: nippleareolar complex; RNSM: Robotic nipple-sparing mastectomy; CNSM: conventional nipple-sparing mastectomy; NRS: numerical rating scale; PONV: postoperative nausea/vomiting; IBR: immediate breast reconstruction; IPBR: immediate prosthesis breast reconstruction; DTI: direct-to-implant; IV-PCA: intravenous patient-controlled analgesia; ADM: acellular dermal matrix; BIS: bispectral index; IV: intravenous.

\section{Acknowledgements}

\section{Ethics Committee Approval and Patient Consent}

This study was approved by the institutional review board and hospital research ethics committee (Yonsei University Health System, Seoul, Korea; IRB protocol No. 4-2020-0991) and was performed following the ethical standards of the current version of the Declaration of Helsinki. As a retrospective study, the requirement for informed consent was waived given that the information and records of the patients were anonymized before analysis.

\section{Authors' contributions}

J.M. and J.L. contributed equally to this work. J.M. and J.L. contributed to study design and conduct, data analysis, and manuscript writing. D.W.L, contributed to methodology, and project administration. H.S.L. contributed to statistical analysis. D.J.N. contributed to investigation. M.J.K. participated in data curation. N.Y.K. and H.S.P. participated as the corresponding author and supervised the overall study and drafting of the manuscript.

\section{Competing Interests}

Jiae Moon, Jeea Lee, Dong Won Lee, Hye Sun
Lee, Hye Ji Joo, and Na Young Kim have no conflicts of interest or financial ties to disclose.

Hyung Seok Park received an honorarium from Intuitive Surgical for his consultation, which is not related to this work.

\section{References}

1. Valero MG, Muhsen S, Moo TA, Zabor EC, Stempel M, Pusic A, et al. Increase in Utilization of Nipple-Sparing Mastectomy for Breast Cancer: Indications, Complications, and Oncologic Outcomes. Ann Surg Oncol 2020; 27(2):344-51.

2. Galimberti V, Vicini E, Corso G, Morigi C, Fontana S, Sacchini V, et al. Nipple-sparing and skin-sparing mastectomy: Review of aims, oncological safety and contraindications. Breast 2017; 34 Suppl 1:S82-S4.

3. Park HS, Lee J, Lee DW, Song SY, Lew DH, Kim SI, et al. Robot-assisted Nipple-sparing Mastectomy with Immediate Breast Reconstruction: An Initial Experience. Sci Rep 2019; 9(1):15669.

4. Sarfati B, Struk S, Leymarie N, Honart JF, Alkhashnam H, Tran de Fremicourt K, et al. Robotic Prophylactic Nipple-Sparing Mastectomy with Immediate Prosthetic Breast Reconstruction: A Prospective Study. Ann Surg Oncol 2018; 25(9):2579-86.

5. Toesca A, Peradze N, Galimberti V, Manconi A, Intra M, Gentilini O, et al. Robotic Nipple-sparing Mastectomy and Immediate Breast Reconstruction With Implant: First Report of Surgical Technique. Ann Surg 2017; 266(2):e28-e30.

6. Selber JC. Robotic Nipple-Sparing Mastectomy: The Next Step in the Evolution of Minimally Invasive Breast Surgery. Ann Surg Oncol 2019; 26(1):10-1.

7. Toesca A, Invento A, Massari G, Girardi A, Peradze N, Lissidini G, et al. Update on the Feasibility and Progress on Robotic Breast Surgery. Ann Surg Oncol 2019; 26(10):3046-51.

8. Lai HW, Chen ST, Mok CW, Lin YJ, Wu HK, Lin SL, et al. Robotic versus conventional nipple sparing mastectomy and immediate gel implant breast reconstruction in the management of breast cancer- A case control comparison study with analysis of clinical outcome, medical cost, and patient-reported cosmetic results. J Plast Reconstr Aesthet Surg 2020. doi:10.1016/

j.bjps.2020.02.021

9. Park HS, Kim JH, Lee DW, Song SY, Park S, Kim SI, et al. Gasless Robot-Assisted Nipple-Sparing Mastectomy: A Case Report. J Breast Cancer 2018; 21(3):334-8.

10. Park HS, Lee J, Lee H, Lee K, Song SY, Toesca A. Development of Robotic Mastectomy Using a Single-Port Surgical Robot System. Journal of Breast Cancer 2020; 23(1):107-12.

11. Lee J, Park HS, Lee H, Lee K, Han DH, Lee DW. Axillary lymph node dissection using a robotic surgical system: Initial experience. J Surg Oncol 2020. doi:10.1002/jso.26141.

12. Ahn SJ, Woo TY, Lee DW, Lew DH, Song SY. Nipple-areolar complex ischemia and necrosis in nipple-sparing mastectomy. Eur J Surg Oncol 2018; 44(8):1170-6.

13. Williamson A, Hoggart B. Pain: a review of three commonly used pain rating scales. J Clin Nurs 2005; 14(7):798-804.

14. Andersen KG, Kehlet $H$. Persistent pain after breast cancer treatment: a critical review of risk factors and strategies for prevention. J Pain 2011; 12(7):725-46.

15. Andersen KG, Duriaud HM, Jensen HE, Kroman N, Kehlet H. Predictive factors for the development of persistent pain after breast cancer surgery. Pain 2015; 156(12):2413-22.

16. Langford DJ, Paul SM, West C, Levine JD, Hamolsky D, Elboim C, et al. Persistent breast pain following breast cancer surgery is associated with persistent sensory changes, pain interference, and functional impairments. J Pain 2014; 15(12):1227-37.

17. Habib AS, Kertai MD, Cooter M, Greenup RA, Hwang S. Risk factors for severe acute pain and persistent pain after surgery for breast cancer: a prospective observational study. Reg Anesth Pain Med 2019; 44(2):192-9.

18. Guru KA, Wilding GE, Piacente P, Thompson J, Deng W, Kim HL, et al. Robot-assisted radical cystectomy versus open radical cystectomy: assessment of postoperative pain. Can J Urol 2007; 14(6):3753-6.

19. Kwon IG, Cho I, Guner A, Choi YY, Shin HB, Kim HI, et al. Minimally invasive surgery for remnant gastric cancer: a comparison with open surgery. Surg Endosc 2014; 28(8):2452-8.

20. Walia GS, Aston J, Bello R, Mackert GA, Pedreira RA, Cho BH, et al. Prepectoral Versus Subpectoral Tissue Expander Placement: A Clinical and Quality of Life Outcomes Study. Plast Reconstr Surg Glob Open 2018; 6(4):e1731. 
21. Li L, Su Y, Xiu B, Huang X, Chi W, Hou J, et al. Comparison of prepectoral and subpectoral breast reconstruction after mastectomies: A systematic review and meta analysis. Eur J Surg Oncol 2019; 45(9):1542-50.

22. Lee J, Park HS, Lee H, Lee DW, Song SY, Lew DH, et al. Post-Operative Complications and Nipple Necrosis Rates between Conventional and Robotic Nipple-Sparing Mastectomy. Front Oncol 2020; 10:594388. 